\title{
KRITISCHE BEMERKUNGEN ZU DEN ARGONAUTICA DES VALERIUS FLACCUS
}

\author{
THOMAS GÄRTNER \\ Universidad de Colonia \\ th-gaertner@gmx.de
}

\section{CRITICAL COMMENTS ON VALERIUS FLACCUS' ARGONAUTICA COMENTARIOS CRÍTICOS SOBRE LAS ARGONÁUTICAS DE VALERIO FLACO}

\begin{abstract}
Auf der Grundlage der modernen Ausgaben und Kommentare wird eine Reihe textkritischer Einzelprobleme aus den Argonautica des Valerius Flaccus besprochen. Es handelt sich um folgende Stellen: I 779-784; II 82-86; II 98-102; II 138-140; III 14-18; III 74-80; III 535-542; IV 150-153; IV 214 f.; IV 438-441; IV 757 f.; V 39 f.; V 182 f.; VI 317-323; VI 683-685; VI 752-760; VII 4-8; VII 121-123; VII 162-165; VII 276-283; VII 635-640; VIII $158 \mathrm{f}$.
\end{abstract}

Stichworte: Valerius Flaccus; Argonautica; flavische Epik; Textkritik.
In the present paper a number of critical problems concerning the Argonautica of Valerius Flaccus are discussed, starting from modern editions and commentaries. Comments are offered on the following passages: I 779-784; II 82-86; II 98-102; II 138-140; III 14-18; III 74-80; III 535-542; IV 150-153; IV 214 f.; IV 438-441; IV 757 f.; V 39 f.; V 182 f.; VI 317-323; VI 683-685; VI 752-760; VII 4-8; VII 121-123; VII 162-165; VII 276-283; VII 635-640; VIII $158 \mathrm{f.}$.

Key words: Valerius Flaccus; Argonautica; Flavian epic; textual criticism.

En este artículo se examina una serie de problemas relativos a la crítica textual de las Argonáuticas de Valerio Flaco a partir de ediciones y comentarios modernos. Se comentan los siguientes pasajes: I 779784; II 82-86; II 98-102; II 138-140; III 14-18; III 74-80; III 535-542; IV 150-153; IV 214 s.; IV 438-441; IV 757 s.; V 39 s.; V 182 s.; VI 317-323; VI 683-685; VI 752-760; VII 4-8; VII 121-123; VII 162-165; VII 276-283; VII 635-640; VIII $158 \mathrm{~s}$.

Palabras clave: Valerio Flaco; Argonáuticas; épica flavia; crítica textual.

I $779-784$

Hunc (sc. taurum) sibi praecipuum gentis de more nefandae

780 Thessalis in seros Ditis seruauerat usus, Tergeminam cum placat eram Stygiasque supremo

Obsecrat igne domos, iamiam exorabile retro

Carmen agens; neque enim ante leues niger auehit umbras

Portitor et cunctae primis stant faucibus Orci. 
Kleywegt z.St. verwirft, ebenso wie jetzt Zissos z.St., im Anschluß an Strand p. 72 sämtliche Versuche, cunctae zu verbessern: functae (Wagner), coetu (Reuss), cinctae (Köstlin) und uinctae (Jacobs, aufgenommen u.a. bei Langen); er argumentiert, daß cunctae «does not here serve to exclude exceptions ${ }^{1}$, but rather insists on their concentrated masses (Pius: agminatim)».

Zunächst erscheint es sprachlich sehr zweifelhaft, ob cunctae im Sinne von densae (was der Dichter ja auch eindeutigerweise hätte schreiben können) verstanden werden kann. Ferner ergibt sich das inhaltliche Problem, daß eine solche Vorstellung «dichtgedrängter Totenschatten» zwar gut in eine Katabasis wie die in Aen. VI beschriebene paßt (hieraus stammen Kleywegts Parallelen), aber weniger in eine gezielte Totenbeschwörung, die sich auf eine Befragung des Cretheus konzentriert (737 f.), also kaum eine unüberschaubar dicht gedrängte Schar von Totenschatten am Eingang der Unterwelt zurücklassen wird.

Es kann kaum ein Zweifel bestehen, daß der herzustellende Ausdruck in der Tat - im Sinne von Jacobs' Versuch uinctae — die Inhibierung der an der Rückkehr in die Unterwelt gehinderten Totenschatten beschreiben muß. Einen passend mit primis ... faucibus Orci kontrastierenden Ausdruck erhält man, wenn man liest

... neque enim ante leues niger auehit umbras

Portitor: exclusae primis stant faucibus Orci.

II $82-86$

Tempore quo primum fremitus insurgere opertos

Caelicolum et regni sensit nouitate tumentes

Iuppiter aetheriae nec stare silentia pacis,

85

Iunonem uolucri primam suspendit Olympo

Horrendum chaos ostendens poenasque barathri.

Den zweimaligen Wechsel der Konstruktion nach sensit mag man hinnehmen, obwohl die bei Poortvliet im Kommentar z.St. zitierten Parallelen I 123 ff. und III 512 ff. nur den Übergang von der geläufigeren Konstruktion

${ }^{1}$ So deutet jetzt wieder Spaltenstein z.St. 
(AcI) zur selteneren (Partizip) belegen. Aber es befremdet, daß das Verbum tumere von den über Jupiters Herrschaft erzürnten Göttern verwendet wird, obwohl es eigentich viel besser auf die Arroganz des neuen Herrschers paßt. Mit leichter Abänderung liest man:

Tempore quo primum fremitus insurgere opertos

Caelicolum $e$ regni sensit nouitate tumentis

Iuppiter aetheriae nec stare silentia pacis,

Mit der Junktur e regni ... nouitate tumentis variiert Valerius Flaccus Verg., Aen. IX 596 tumidusque nouo praecordia regno.

II $98-102$

... contra Veneris stat frigida semper Ara loco, meritas postquam dea coniugis iras

100 Horruit et tacitae Martem tenuere catenae.

Quocirca struit illa nefas Lemnoque merenti Exitium furiale mouet ...

Wenn der Erzähler die Bestrafung der Venus für ihren Ehebruch als 'verdient' betrachtet (meritas ... coniugis iras), so wundert man sich, daß er nicht auch die hieraus resultierende Verödung der lemnischen Altäre der Liebesgöttin als berechtigt ansieht, sondern im Gegenteil auch die Bestrafung von Lemnos hierfür als verdient bezeichnet (Lemnoque merenti / Exitium furiale mouet), noch dazu unter Verwendung desselben Verbs. Eine solche Inkonsequenz des Erzählstandpunkts muß befremden, auch wenn man meritas in dem neutralen Sinne von 'incurred' zu deuten versucht (Poortvliet z.St.) und merenti nur «ex sententia Veneris» (Langen z.St., anders Spaltenstein z.St.) verstehen möchte. Offenbar hat man es bei merenti mit einem Influenzfehler zu tun, der mit trementi leicht rückgängig gemacht werden kann, vgl. II 196-198 Ipsa Venus quassans undantem turbine pinum / Agglomerat tenebras pugnaeque accincta trementem / Desilit in Lemnon. 
II $138-140$

... manet illa (sc. Eurynome) uiro famulasque fatigat

Litoribus, tardi reputant quae tempora belli

140 Ante torum et longo mulcent insomnia penso.

Schwierigkeiten bereitet die Junktur fatigat / Litoribus (138 f.). Die Verteidiger der Überlieferung verstehen den Ausdruck derart, daß die treue Eurynome ihre Dienerinnnen mit der Überwachung des Strands beauftragt (Vossius: «Eurynomen ... fatigare famulas discursitando per littora», Heinsius «cum interim famulas iterum iterumque mittendo ad litus fatigaret», Thilo proleg. xvi «ab Eurynome ... famulas fatigatas esse iterum iterumque ad litora missas», Kramer im Apparat z.St. «litoribus intellego iteratis ad litus itionibus», Liberman «et épuise à surveiller le rivage ses servantes»; Dräger «ermüdet ihre Mägde / durch die 〈Beobachtung der〉 Gestade»). Spaltenstein sucht die Überlieferung anders durch eine etwas erweiterte Bedeutung von Litoribus zu erklären (litora $=$ spes, quae in litoribus ponitur) und verweist auf Sil., Pun. II 459-461 (den Bewohnern des von den Carthagern belagerten Sagunt schwindet die Hoffnung auf römische Unterstützung):

... tandem aequore uano

460 Auertunt oculos frustrataque litora ponunt

Et propius suprema uident ...

(damnant für ponunt erwägt Delz 1997, S. 165). Andererseits findet sich schon in der Editio Aldina die Konjektur Velleribus, die von Poortvliet im Kommentar z.St. (vgl. jetzt auch 2001, S. 353) durch Muneribus modifiziert wird.

Unterblieben ist bislang, soweit ich sehe, der Versuch, die Überlieferung mit einer neuen Interpunktion beizubehalten: Setzt man das Komma vor statt hinter Litoribus, so erhält Ante torum mit dem dann lokativen Litoribus ein exaktes, gleichfalls betont an die Satzspitze gerücktes Pendant, und Eurynome «ermüdet» ihre Dienerinnen auf zweifache Weise: Sie läßt diese einerseits (tagsüber) am Strand (dem Ort der Abreise und der erhofften Rückkehr des Heers) die Dauer des sich hinziehenden Krieges berechnen, andererseits (nachts) im Schlafgemach sich über ihre Schlaflosigkeit mit langdauernden Wollarbeiten hinwegtrösten. 
III $14-18$

Tu mihi nunc causas infandaque proelia, Clio,

15 Pande uirum! tibi enim superum data, uirgo, facultas

Nosse animos rerumque uias. cur talia passus

Arma, quid hospitiis iunctas concurrere dextras

Iuppiter? unde tubae nocturnaque mouit Erinys?

Der Schlußsatz des Binnenprooemiums ist in seiner grammatikalischen Konstruktion durchaus problematisch: Langen faßt nocturna als Neutrum Plural und sieht darin das Objekt zu mouit (vgl. Dräger: «Woher 〈kamen〉 die Kriegstrompeten und erregte nächtliche Ereignisse die Erinye?»), wogegen Shackleton Bailey 1977, S. 203 protestiert, der mit mugit statt mouet einen Ausdruck herstellt, der zeugmatisch sowohl auf tubae als auch auf nocturna ... Erinys paßt (Spaltenstein hilft sich dagegen, indem er mouit intransitiv versteht). Allerdings erwartet man eher ein Perfekt als ein Präsens, und das überlieferte Perfekt mouit («setzte in Bewegung») scheint in einem Ursachenabschnitt sehr passend.

Genaugenommen steht die nocturna ... Erinys als potentielle Verursacherin auf einer anderen Ebene als die (metonymisch für den Kampf stehenden) tubae. Insofern kann man mit sehr geringem Eingriff schreiben:

... unde tubae? nocturna ne mouit (sc. tubas) Erinys?

III $74-80$

At Minyas anceps fixit pauor; aegra uirorum

75 Corda labant, nec quae regio aut discrimina cernunt, Cur galeae clipeique micent, num peruigil armis

Donec et hasta uolans immani turbine transtris Insonuit monuitque ratem rapere obuia caeca

80 Arma manu ...

Im codex Carrionis sind nach Vers 76 die Worte Hostis et exciti dent obuia praelia Colchi nachgetragen. Dieser Vers wirkt mit seiner hölzernen Juxtaposition Hostis et ... Colchi wie ein künstlicher Versuch, eine vorgegebene Lücke auszufüllen. Sachlich ist die Annahme, man werde bereits in 
diesem Stadium der Reise von den Kolchern angegriffen, absurd und scheint auf einem Missverständnis von Jasons Aufforderung in III 82 (Vosque, uiri, optatos huc adfore credite Colchos) zu beruhen, vgl. Hurka 2003, SS. 39-43. Andererseits kann man aber kaum nach peruigil armis einfach die Worte hostis sit supplieren, wie Ehlers will (vgl. 1976, S. 259), der den Text ohne Lücke druckt (Hurka 2003, S. 42 f. kehrt wieder zur Annahme einer Lücke zurück).

Die Frage num peruigil armis bringt nichts wesentlich Neues zum Ausdruck, insofern ja nach galeae clipeique im Zusammenhang klar ist, daß es sich um nächtliche Waffen handelt. Man könnte ohne Lücke auskommen und zugleich einen gedanklichen Fortschritt gegenüber Cur galeae clipeique micent erzielen, indem man armis als Minuskelkorruptel aus astus ansieht (zur Korruptel vgl. Sil., Pun. III 124 tremor implicat artus [ar(c)tus FOr1 : armis Lb]): «ob es sich um eine nächtliche Kriegslist handelt». peruigil astus ist dann eine erlesene dichterische Variante zu nocturnus dolus, vgl. Coripp., Ioh. IV 412 multo peruigil astu und Radulf. Cad., Gest. Tancr. XXX 8-11 (RHC III 627 FG):

Dux ergo Turcas acies premit, insequiturque

Non astu uigili, non commoditate locali,

Non quae uel timidos in fortes excitat, arte

Adiutus.

III 535-542 (Juno über Hylas zur Nymphe Dryope)

535 Quem tibi coniugio tot dedignata dicaui, Nympha, procos, en Haemonia puer appulit alno, Clarus Hylas, saltusque tuos montesque pererrat. Vidisti roseis haec per loca Bacchus habenis Cum domitas acies et eoi fercula regni

540 Duceret ac rursus thiasos et sacra mouentem. Hunc tibi uel posito uenantem pectine Phoebum Crede dari ...

Abgesehen von der Inkonzinnität der Konstruktion nach Vidisti (Pius änderte mouentem in moueret) befremdet der motivische Ablauf: Dryope soll Bacchus nach seinem indischen Erorberungszug noch ein weiteres Mal gesehen haben, als er nurmehr seinen Kult veranstaltete («vidit Dryope Bacchum 
bellatorem et victorem redeuntem, tum postea eum thiasos et sacra moventem», Langen). Anstatt dieses antiklimaktischen Ablaufs wird man mouentem besser als ein Hunc sc. Bacchum modifizierendes und mit uenantem ... Phoebum korrespondierendes Partizip zur folgenden Periode ziehen:

Vidistis roseis haec per loca Bacchus habenis

Cum domitas acies et eoi fercula regni

540 Duceret; $\langle\mathrm{h}>$ ac rursus thiasos et sacra mouentem

Hunc tibi uel posito uenantem pectine Phoebum

Crede dari ...

Erst so gewinnt rursus («jetzt erstmals wieder nach dem indischen Feldzug») befriedigenden Sinn. 〈h〉ac rursus greift auf haec per loca zurück.

IV $150-153$

Talis in aduectos Neptuni credita proles

Aeternum furit, atque aequae uirtutis egentes

Ceu superum segnes ad iniqua altaria tauros

Constituit, tandem ut misero lauet arma cerebro.

Die Logik des $u t$-Satzes in V. 153 bleibt problematisch. Gemäß IV $109 \mathrm{ff}$. fordert Amycus nur die Stärkeren unter seinen «Gästen» zum Boxkampf heraus, während er die Schwächeren gleich opfert. Insofern misero lauet arma cerebro offenkundig von den Opfern des Boxkampfs gesagt wird, scheint im $u t$-Satz von einer anderen Personengruppe als im Hauptsatz die Rede zu sein. An dieser Problematik ändern weder die Konjekturen von Burmann (tantum für tandem), Löhbach (cruda für tandem) oder Heinsius (sanie ac misero ut lauet) etwas, die nur das unverständliche tandem beseitigen, noch das Verfahren von Liberman, der tandem mit Constituit verbindet.

Das eigentliche Problem der Partie wird von Strand 96 f. berührt, der tandem in saltem ändert, und glaubt, Amycus gehe bei seinen schwächeren Opfern einer Art «Ersatzbefriedigung» nach, die ihn an den Boxkampf erinnert:

... in the case of the weaklings Amycus cannot enjoy the pleasure of a regular combat, but still, cruel and bloodthirsty as he is, he uses his caestus when killing them in order at least to soak them with the smashed brains of his poor victims. 
Selbst wenn man diese Vorstellung sachlich akzeptiert, so hätte Valerius Flaccus sie mit dem Satz «er stellt die Schwachen wie Opfertiere an den Altar, um wenigsten mit ihrem unglücklichen Gehirn seine Waffen zu tränken» reichlich unverständlich ausgedrückt; insbesondere fehlt der entscheidende, von Strand supplierte Gedanke «he uses his caestus when killing them».

Gegen Strands Deutung wendet sich Venini 1972, SS. 404-406, die —wie Korn und jetzt Murgatroyd z.St., die sich beide Venini anschließen- die gesamte Partie im Gegensatz zu Strand nicht auf die Geopferten, sondern auf die im Boxkampf Getöteten beziehen will: Venini hält tandem, beruft sich auf die Doppeldbedeutung von constituere, was nicht nur eine «Aufstellung zum Opfer», sondern auch eine «Aufstellung zum Kampf» bezeichnen kann, versteht den gesamten V. 152 als bloßen Vergleich und deutet:

Er stellt seine (allesamt) ungleichen Gegner im Boxkampf auf, wie es mit untätigen Stieren an Götteraltären geschieht, um schließlich seine Handschuhe im unglücklichen Gehirn (der Gegner) zu baden.

Wenn Valerius Flaccus dies hätte sagen wollen, hätte er freilich unsägliche Verwirrung angerichtet mit den Worten Ceu superum segnes ad iniqua altaria tauros / Constituit, die jedermann nach V. $109 \mathrm{ff}$. von einem eigentlichen Opfer verstehen wird, und es insbesondere versäumt, Constituit durch einen Zusatz in eindeutiger Weise zu erweitern.

Es bleibt dabei, daß im Finalsatz von einer anderen Kategorie von Opfern des Amycus die Rede sein muß als im Hauptsatz. Dieser Finalsatz gewinnt seinen Sinn erst zurück, wenn der Bezug auf eine neue Personengruppe durch einen mit misero ... cerebro zu verbindenden Genitiv Plural deutlich wird: Amycus stellt (nur) die Schwachen wie Opfertiere an den Altar, um mit dem unglücklichen Gehirn der Stärkeren seine Waffen tränken zu können. Diese Stärkeren sollten mit einem zu aequae uirtutis egentes kontrastierenden Ausdruck als dem Amycus «Gleichstarke» bezeichnet werden:

... atque aequae uirtutis egentes

Ceu superum segnes ad iniqua altaria tauros

Constituit, parium ut misero lauet arma cerebro.

Zur Verschleifung vgl. V 600 et uocem paria arma sequuntur. 
IV $214 \mathrm{f}$.

Iam pridem caestus resides et frigida raris

215 Dentibus aret humus. quis mecum foedera iunget?

Der Begriff 'ist trocken' (aret) paßt nicht zu Dentibus. Korn z.St. versucht den Begriff 'Blut' in seine umständliche Paraphrase einzuführen («humus arida frigidaque est, quod diu sanguine caesorum non incaluit, et pauci dentes excussi humi iacent»; Spaltenstein spricht von «ingeniöser Elliptik» des Valerius Flaccus); andererseits obelisiert Liberman Dentibus und erwägt im Apparat Caedibus. Aber Dentibus ist beizubehalten, weil der Verlust von Zähnen weitaus charakteristischer für den Boxkampf ist als das Vergießen von Blut. Vielmehr muß der Verbalbegriff an Dentibus angepaßt werden. Wahrscheinlich ist aret nur ein Versuch, die Variante arcet zu verbessern, die aus ursprünglichem albet entstand.

IV 438-441 (Rede des Phineus an die Argonauten)
Nouimus et diuis geniti quibus et uia iussos
Quae ferat ac uestri rebar sic tempora cursus
440 Proxima quaeque legens, quantum Vulcania Lemnos
Traxerit, infelix tulerit quae Cyzicus arma.

Was Phineus berechnete (rebar), war zunächst einmal, wie lange Lemnos die Argonauten aufhielt (quantum Vulcania Lemnos / Traxerit). Der zweite Punkt seiner Rechnung bestand aber nicht darin «welche Waffen der unglückliche Cyzicus ertragen hat», sondern vielmehr, «wielange der Nachtkampf gegen Cyzicus die Argonauten aufhielt»: infelix tulit et quae Cyzicus arma sc. quantum traxerint.

IV 757 f. (Jason stellt dem Lycus den Bezwinger des Amycus vor)

Fatur, et ostentans prolem Iouis 'hic tibi Pollux

En' ait, 'inuiso soluit cui pectore poenas'.

Columbus stellte mit inliso bzw. eliso ... pectore ebenso wie Bulaeus mit inciso ... pectore die Schilderung einer Brustverletzung her, welche nicht recht zum Boxkampf passen will; Schenkl gewann mit seiner von Langen 
und Liberman akzeptierten Konjektur inuito ... pectore eine Trivialität (vgl. jetzt Murgatroyd z.St.); was mit dem von Courtney erwogenen inuiso ... corpore gewonnen würde, ist nicht einzusehen.

Man erwartet einen Hinweis auf das Wie von Amyclus' Tod, der von Pollux gerade in der von ihm bevorzugten Kampfart getötet wurde (vgl. $753 \mathrm{f}$. lege quod occidit ultus / Ipse sua), nämlich im (seinen Opfern) verhaßten Boxkampf. Für pectore wird man also ein Requisit des Boxkampfs herstellen: inuiso soluit cui tergore poenas, vgl. OLD s.u. tergum 7 b und Val. Flacc., Arg. I 420 f. Taurea uulnifico portat celer aspera plumbo / Terga Lacon und zur Nebenform tergus, tergoris Arg. I 130. Zum Motiv vgl. Sil., Pun. X 562 f. expertis inuisus et ensis / Et clipeus.

V $39 \mathrm{f}$.

Bina (nefas) toto pariter mihi funera surgunt

40

Litore; magna adeo comitum numerosaque pubes?

Strand 1972, S. 105 f. und nach ihm Wijsman und Spaltenstein z.St. verteidigen das überlieferte toto gegen Sabellicus' Konjektur tuto, aber der Vergleich mit Stellen wie Verg., Aen. XI 199 f. tum litore toto / Ardentis spectant socios (Spaltenstein) und Luc., Phars. IX 180 f. et toto litore busta / Surgunt Thessalicis reddentia manibus ignem (von Wijsman als Vorbild betrachtet) zeigt nur, wie lächerlich die Verwendung von toto ... / Litore in bezug auf nur z w e i Begräbnisse ist. In der Aussage «at the same time I see (not one but) two funerals fill the whole shore» (Strand) wäre genaugenommen nur eine übertriebene lokale Ausdehnung der beiden funera impliziert.

Das eigentlich Betrübliche für Jason liegt hingegen darin, daß er an d e m s elben Strand zwei Gefährten zugleich verliert. Man wird eine geläufige numerische Antithese herstellen:

Bina (nefas) uno pariter mihi funera surgunt Litore ...

V $182 \mathrm{f}$.

... simul aethere plena corusco

Pallas et alipedum Iuno iuga sistit equorum. 
Wijsman plädiert für Köstlins Herstellung von frena für plena, die auch Delz in Ratis omnia uincet (Spudasmata 48) 12 empfahl. Aber eher als ein Synonym für «Wagen» erwartet man ein Partizip, welches den separativen Ablativ aethere ... corusco stützt (Columbus wollte prona) und sich zugleich mit simul verbindet: simul aethere lapsa corusco, vgl. Arg. V 253 lapsus montibus anguis; $638 \mathrm{f}$. Vel tu sola (sc. Pallas) polo tacitis inopina tenebris / Labere; von Juno und Minerva auch in Arg. I 91 f. Accepere deae celerique per aethera lapsu / Diuersas petiere uias.

VI 317-323

Te quoque, Canthe, tui non inscia funeris Argo

Fleuit ab inuita rapientem tela carina.

Iam Scythicos miserande sinus, iam Phasidis amnem

320 Contigeras, nec longa dies ut capta uideres

Vellera et Euboicis patrios de montibus ignes.

Illum ubi congressu subiit Gesander iniquo

Territat his: '... / ...'

Das distanzierende Personalpronomen Illum (322) knüpft sehr hart und unmittelbar an die programmatisch mit Te quoque begonnene und mit Contigeras bzw. uideres fortgeführte Apostrophe an. Größere formale Geschlossenheit gewinnt der Abschluß, wenn an den in Vv. 319-321 fixierten Zeitpunkt innerhalb von Canthus' Lebenslauf (nach Erreichung des erstrebten Ziels, kurz vor der erhofften Rückkehr) angeknüpft wird:

Tunc ubi congressu subiit Gesander iniquo

Territat his: '... / ...'

Absolutes subire von feindlicher Annäherung wie in Arg. II 519 ingentique umbra subit.

VI 683-685

At quotiens uis dura ducum densique repente

Aesoniden pressere uiri cumque omnis in unum

685 Imber iit, totiens saxis pulsatur (sc. Medea) et hastis. 
Die gestörte totiens-quotiens-Relation (Baier z.St. spricht von «leichter Inkonzinnität») wird man nicht durch Friesemanns bei Thilo referierte Konjektur ceuque omnis wiederherstellen, sondern durch ein die Zuspitzung bezeichnendes iamque omnis.

VI $752-760$

Nox simul astriferas profert optabilis umbras

Et cadit extemplo belli fragor aegraque muris

Digreditur longum uirgo perpessa timorem.

755 Ut fera Nyctelii paulum per sacra resistunt,

Mox rapuere deum iamiam sin quodcumque paratae

Thyiades, haud alio remeat Medea tumultu

Atque inter Graiumque acies patriasque phalangas

Semper inexpletis agnoscit Iasona curis

760 Armaque quique caua superest de casside uultus.

Die Vorstellung, daß die Bakchantinnen während der Kultfeier «einhalten» (per sacra resistunt), ergibt keinen Sinn. Man wird den eigentlichen Vergleichssatz (Vv. 755-757 a) enger mit dem Vorigen verbinden und per sacra in post sacra ändern: Die Bakchantinnen halten $\mathrm{nach}$ der eigentlichen Kultfeier ein wenig ein, um dann später den Gott völlig in sich aufzunehmen (rapuere deum). Im Falle Medeas entspricht ihre Betrachtung des Kampfgeschehens auf der Stadtmauer der eigentlichen kultischen Initiation, der hier geschilderte Moment ihres Weggehens dem zeitweiligen Nachlassen des Enthusiasmos unmittelbar nach der Initiation, und ihre späteren Taten dem mit Mox beschriebenen späteren Gehabe der Bakchantinnen. per sacra läßt sich leicht als Influenzfehler aus dem darüberstehen perpessa erklären, und post sacra entspricht auf der Gleichnisebene exakt dem Partizip perpessa timorem (sc. während der Mauerschau).

VII 4-8

Ergo ubi cunctatis extremo in limine plantis

5 Contigit aegra toros et mens incensa tenebris

Vertere tunc uarios per longa insomnia questus

Nec pereat quo scire malo, tandemque fateri

Ausa sibi paulum medio sic fata dolore est: / ... 
Das von Ehlers und Spaltenstein obelisierte paulum verbindet sich sinnvoll weder mit fateri (Stadler: «Medea schreckt vor der ganzen Wahrheit zurück»; Dräger: «nachdem sie es gewagt hatte, es sich ein wenig einzugestehen») noch mit fata (Burmann, der VII 384 paulumque his uocibus haesit verglich). Perutelli 1992, 229-231 und im Kommentar (Florenz, 1997) z.St. will paulum ebenfalls mit fata verbinden und fügt zu der von Burmann verglichenen Stelle noch Stat. Theb. VII 290 hinzu: Dixerat, et paulum uirgo interfata loquenti. Aber bei Medeas folgendem Monolog handelt es sich weder um ein «kurzes Zögern» noch um eine «kurze Einrede»; mit paulum ... sic fata («sprach ein wenig folgendermaßen») würde das vorausgehende die Bedeutung der Rede ins Licht setzende Partizipialglied tamdemque fateri / Ausa sibi geradezu nachträglich lächerlich gemacht und die Bedeutung von Medeas folgendem Monolog minimalisiert.

Heinsius änderte paulum in pauidum (inneres Objekt zu fata) und verglich Ou., Met. IX 569 Et pauidum (v.l. paulum) blandita; Schenkl variierte dies zu pauida in. Aber eher erwartet man ein die «Liebe» mit negativer Konnotation umschreibendes und malo bzw. dolore variierendes Objekt zu fateri. Gegenüber Wagners recht abstraktem causam (akzeptiert bei Langen und Liberman, Courtney will culpam, Watt 1984, S. 167 praeferiert uerum, Delz in Ratis omnia uincet 16 planum als - unbelegtes - inneres Objekt zu fateri) empfiehlt sich ein die Liebe idiomatisch umschreibendes pestem, vgl.:

Talia uerba dabat conlapsaque flebat iniquae in Veneris Medea sinus pestemque latentem

Ossibus atque imi monstrabat pectoris ignem (VII 251-253)

cur tanta mihi (sc. matri) non prodita pestis (VIII 165);

sonst etwa Verg. Aen. I 712; IV 90; Cat. 76, 20.

VII 121-123

Tum comitum uisu fruitur miseranda suarum Implerique nequit subitoque parentibus haeret Blandior et patriae circumfert oscula dextrae.

Die singuläre Junktur patriae circumfert oscula dextrae (Spaltenstein hebt hervor, daß circumferre üblicherweise mit konkreten Objekten verbunden 
wird; laut Perutelli malt das Kompositum «la frenetica agitazione della fanciulla») müßte man mit Langen in dem Sinne «oscula fert in omnes dextrae partes» deuten; mit dem bei Stadler z.St. zitierten Perkins die Wahl des Kompositums circumfert auf eine Korrespondenz mit dem «in religöser Terminologie synonymen» Verb lustrat zurückzuführen, das im folgenden Gleichnis in V. 126 gebraucht wird, geht kaum an, zumal beide Verben hier ganz abseits der religiösen Terminologie verwendet werden. Gegenüber Heinsius' Konjekturen nunc offert und rursum fert empfiehlt sich als einfacherer Eingriff eher ein seinerseits pathetisch intensivierendes patriae centum fert oscula dextrae (vgl. etwa Cat., Carm. 5.7 Da mi basia mille, deinde centum).

VII 162-165 (Juno zu Venus)

I precor atque istum quo me frustratur amorem Vince $†$ precor $†$ patriis ut tandem euadere tectis Audeat atque meum casu defendere ab omni 165 Aesoniden ...

Der Begriff amorem ist auf zweifache Weise gedeutet worden.

Einerseits verstand man ihn als Ausdruck der sexuellen Liebe Medeas zu Jasons. Diese Deutung bedingt, daß man uince in der praegnanten Bedeutung 'erzeuge siegreich' verstehen muß (so Langen, Stadler, Perutelli, Spaltenstein z.St. und Hurka 2003, S. 119: 'setze siegreich durch'). Dann wird man für das evidentermaßen auf einem Influenzfehler aus V. 162 beruhende zweite precor Entsprechendes einsetzen: Baehrens wollte tori, Stadler e.g. uiri oder ducis (sc. Iasonis). Diskreditiert wird eine solche Deutung aber durch den auf amorem bezüglichen Relativsatz quo me frustratur: Wie kann Juno sagen, Medea täusche sie durch die (von Juno selbst mit Hilfe der Venus gestiftete) Liebe zu Jason (vgl. Dräger: «verhilf dieser Liebe, mit der sie mich hinhält, / Zum Sieg»)? Spaltenstein versucht im Anschluß an Langen die Deutung «die Liebe, um welche (d.i. um deren Verwirklichung) Medea mich betrügt» — was den grotesken Eindruck vermitteln könnte, Juno wäre selbst verliebt.

Bei dieser Deutung wäre es auch möglich, für das zweite precor etwas anderes als ein Attribut oder einen attributiven Ausdruck zu amorem einzusetzen: Liberman stellt parenthetisches potes her, Ehlers erwägt soror (was wohl eine freundschaftliche, nicht-genealogische Anrede der Juno an Venus sein soll), Taliercio vokativisches Venus, Sandstroem schrieb (unter der Annahme, 
etwas paläographisch Ähnliches wäre einzusetzen) procax, was dieser selbst auf Vince, Langen hingegen auf Audeat bezog.

Andererseits hat man hinter amorem auch das Gegenteil dessen gesucht, was das Wort auf den ersten Blick zu bedeuten scheint, nämlich die Bindung Medeas an ihre Heimat und ihr Elternhaus. Dann kann uince seine triviale Bedeutung 'besiege' behalten, aber amorem benötigt dringend eine in diese Richtung gehende nähere Bestimmung: Loehbach änderte precor in patris (was Stadler z.St. zurecht schon wegen des Nebeneinanders von patris und patriis ablehnt), und Burmann änderte gar mit hartem Eingreif zu istum quo me frustrata pudorem, / Vince prior.

Der Relativsatz quo me frustratur entscheidet zugunsten der letzteren Deutung (damit scheitert automatisch der Versuch, das zweite precor zu halten, von Poortvliet 2003, S. 607): Medea täuscht Junos Erwartungen durch ihre unerwartet starke familiäre Bindung. Die amoralische Paradoxie von Junos Bestreben (vgl. das deutlich abwertende Pronomen istum) tritt dann richtig hervor, wenn man amorem mit einem moralisierenden (und zugleich den Inhalt des Substantivs eindeutig klärenden) Attribut verbindet:

I precor atque istum quo me frustratur amorem Vince pium, patriis ut tandem euadere tectis Audeat atque meum casu defendere ab omni

166 Aesoniden ...

VII 276-283

Si Pelopis duros prior Hippodamia labores Expediit totque ora simul uulgata procorum Respiciens tandem patrios exhorruit axes, Si dedit ipsa neci fratrem Minoia uirgo,

280 Cur non hospitibus fas sit succurrere dignis

Te quoque et Aeaeos iubeas mitescere campos?

Occidat aeterna tandem Cadmeia morte

Iam seges et uiso fumantes hospite tauri.

Thilo versetzte die Versreihe an das Ende von Venus' Trugrede hinter V. 291, insofern sich die unmittelbare Anrede an Medea in V. 280 f. nicht in das Referat einer angeblich von Jason an Kirke gerichteten Rede (in welcher Kirke angesprochen, aber von Medea in der dritten Person geprochen wird) 
paßt. Die meisten späteren Herausgeber folgten Thilo; Kramer vermutete eine auktoriale Alternativfassung für den überlieferten Epilog der Kirke-Rede (V. 288-291). Über die Qualität der vermeintlichen Doppelfassungen gelangten Kramer und Jachmann zu bedenklich konträren Auffassungen (vgl. W.W. Ehlers 1985, S. 347 f.).

Thilos Versetzung führt jedoch zu erheblichen Problemen: Zum einen raubt sie der Trugrede der Venus eine brilliante Schlußpointe, die darin besteht, daß Kirke am Schluß nicht mehr unmittelbar an das Mitleid der Medea mit Jason apelliert, sondern an deren Ehrgeiz als Zauberin.

Bereits Kramer führte gegen Thilo ins Feld, daß die Rede offensichtlich mit V. 291 enden müsse. Hiergegen Ehlers a.a.O. 348 ff., der aber nur zeigt, daß sich aus dem Ganzen «kein Teil herauslösen» läßt, also nichts ausgeschieden werden darf; die Tatsache, daß V. 290 f. als persönlicher Schluß der Worte der vermeintlichen Kirke an Medea viel besser passen als das nach der Umstellung am Redeende stehende mythische Paradigma, wird von Ehlers ignoriert.

Eine zweite Schwierigkeit liegt darin, daß das Ariadne-Exempel auch bei Apollonius Rhodius (III 997 ff.) von Jason verwendet wird; insofern ist es durchaus problematisch, dieses Exempel bei Valerius Flaccus infolge einer Konjektur einer anderen Person in den Mund zu legen, zumal die sich passend an die Vorstellung einer erfolgreichen Drachensaat Jasons anknüpfenden folgenden Worte (284 Ei mihi, quod nullas hic possum exsoluere grates) evidenterweise die vergleichsweise selbstbewußtere Dankankündigung

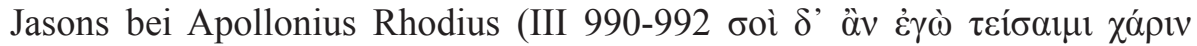

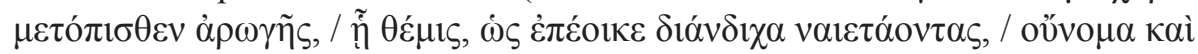

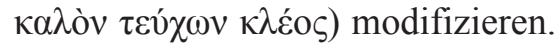

Also spricht alles dafür, daß auch bei Valerius Flaccus Jason das Ariadne-Exempel bemüht und daß die Verse 276-281 an der richtigen Stelle überliefert sind. Die direkte Anrede an Medea wird auf der Verschlimmbesserung eines Kopisten beruhen, der nicht begriff, daß es sich um eine referierte Rede Jasons, die sich unmittelbar an Kirke und nicht an Medea richtet, handelte. Eine «unpersönliche» Form der V. 280 f. läßt sich mit Hilfe leichter Eingriffe zurückgewinnen:

Cur non hospitibus fas sit succurrere dignis

Nunc quoque et Aeaeos pudeat (subeas codd.) mitescere campos?

Jason fragt: «Warum sollte eine solche Hilfsaktion nicht auch hier und heute möglich sein?». Nunc quoque knüpft an Si ... prior (276) an. Mit Ae- 
aeos pudeat mitescere campos vgl. Stat., Achill. I 272 pudet hoc mitescere cultu? An den Wunsch, die Felder von Aea mögen ihrerseits «mild werden», fügen sich die Verse $282 \mathrm{f}$. konkretisierend an. Schließlich erscheint Cur non ... fas sit ... et (sc. cur) pudeat ... sprachlich glatter als in der überlieferten Fassung Cur non ... fas sit ... et (sc. cur non) iubeas, wo man strenggenommen statt et ein nec erwarten müßte.

VII 635-640

635 Qualis ubi atto〈nitos〉 maestae Phrygas annua Matris

Ira uel exsectos lacerat Bellona comatos, Haud secus accensas subito Medea cohortes Implicat et miseros agit in sua proelia fratres. Omnis ibi Aesoniden sterni putat, omnibus ira 640 Talis erat ...

Talis erat wurde vielfach abgeändert (meist mit dem Argument, es könne sich nicht mehr auf den Vergleich zurückbeziehen), in Aequalis (Ehlers 1971-1972, 132 nach Heinsius, der irae / Aequales wollte), in Letifera est (Courtney 1965, 155), in Falsa errat (Madvig), in Letalis (Watt) oder in Fatalis (Erwägung von Liberman). Stadler im Kommentar (Spudasmata 49) z.St. erkennt dagegen richtig (anders Perutelli z.St.), daß das Kolon omnibus ira/ Talis erat nichts Neues besagt, sondern nur der Bekräftigung des vorausgehenden Omnis ibi Aesoniden sterni putat dient: omnibus ira talis erat quasi Aesonides ibi sterneretur (Spaltensteins Deutung talis erat $\mathrm{sc}$. qualis ceteris wäre trivial), also daß die Überlieferung in V. 640 nicht anzutasten ist.

Allerdings ist der Rückbezug von ibi (i.e. in proeliis suis) nicht gerade luzide, und omnibus ira / Talis erat würde noch leichter verständlich, wenn jeder einzelne Teilnehmer am «Bruderkampf» glaubt, Jason werde von ihm getötet. Daher wird man das singularische Omnis i.q. 'jedermann' für eine Korruptel ansehen und überliefertes Omnis ubi (ibi Carrio) anders berichtigen:

\section{Quisque sibi Aesoniden sterni putat: omnibus ira \\ 614 Talis erat ...}

Vgl. VI 350 f. armaque Canthi / Quisque sibi et Graio poenam de corpore poscens. 
VIII 158 f. (Rede von Medeas Mutter)

Sed quid ego quemquam immeritis incuso querellis? Ipsa fugit tantoque (nefas) ipsa ardet amore.

Courtney (1961, S. 107) schreibt Sed quid ago quemque nach dem Vorgang von Columbus (ago quemue). Das Verbum ago bietet sicher eine inhaltlich gegenüber Lucian Müllers ego $\langle$ 〉 quemquam (aufgenommen bei Liberman) überzeugendere Beseitigung der verdächtigen jambischen Messung von ego (gemäß ThLL V 2, 252, 80 ff. sehr selten in klassischer daktylischer Dichtung), aber das überlieferte quemquam ist nicht anzutasten. Wenn man schreibt

Sed quid ago? quemquam immeritis incuso querellis?

Ipsa fugit tantoque (nefas) ipsa ardet amore,

so ist quemquam in durchaus normgrammatischer Weise (anders als in der Überlieferung) in einer rhetorischen Frage mit negativer Aussagetendenz verwendet (num quemquam immeritis incuso querellis?). Diese sachlich auf «ich will keinen anderen unverdient anklagen» hinauslaufende Aussage präpariert denkbar wirkungsvoll das nachfolgende Ipsa fugit.

Ein exakt paralleles Textproblem ergibt sich in Ou., Epist. 13.133: Sed quid ago (v.l. ego)? reuoco? reuocaminis omen abesto.

\section{LITERATURVERZEICHNIS}

Baier, Th. 2001: Valerius Flaccus, Argonautica Buch VI. Einleitung und Kommentar, München.

Courtney, E. 1961: «On Valerius Flaccus», $C R$ 75, p. $106 \mathrm{f}$.

- 1965: «Valeriana tertia», CR 79, 1965, pp. 151-155.

- 1970: C. Valerii Flacci Argonauticon libri octo, Leipzig.

Delz, J. 1997: «Nachlese zu Silius Italicus», MH 54, pp. 163-174.

Dräger, P. 2003: C. Valerius Flaccus, Argonautica, Frankfurt.

Ehlers, W. W. 1976: Rezension zu Courtney 1970, Gn. 48, 1976, pp. 255-260.

- 1971-1972: «Valerius Flaccus 1940-1971», Lustrum 16, pp. 105-142.

- 1980: Gai Valerii Flacci Argonauticon libri octo, Stuttgart.

— 1985: «Valerius-Probleme», MH 42, 1985, pp. 334-350.

Hurka, Fl. 2003: Textkritische Studien zu Valerius Flaccus, Hermes Einzelschriften 90, Stuttgart. 
Kleywegt, A. J. 2005: Valerius Flaccus, Argonautica, Book I, A Commentary. Leiden.

Korn, M. 1989: Valerius Flaccus, Argonautica 4, 1-343. Ein Kommentar, Hildesheim.

Kramer, O. 1913: C. Valerii Flacci Sentini Balbi Argonauticon libri octo, Leipzig.

Langen, P. 1896 f.: C. Valeri Flacci Sentini Balbi Argonauticon libri octo, Berlin.

Liberman, G. 1997/2002: Valerius Flaccus, Argonautiques, t. 1: Chants I-IV; t. II: Chants V-VIII, Paris.

Murgatroyd, P. 2009: A Commentary on Book 4 of Valerius Flaccus' Argonautica, Leiden.

Perutelli, A. 1992: «Due presunte corruttele nel libro VII di Valerio Flacco», MD 28, pp. 229-231.

- 1997: C. Valeri Flacci Argonauticon Liber VII, Florenz.

Poortvliet, H. M. 1991: C. Valerius Flaccus Argonautica Book II, Amsterdam.

- 2001: Rezension zu Liberman 1997, Mnem. 54, pp. 350-356.

— 2003: «Valerian Leftovers (III)», Mnem. 56, pp. 606-610.

Shackleton Bailey, D. R. 1977: «On Valerius Flaccus», HSPh 81, pp. 199-215.

Spaltenstein, F. 2002: Commentaire des Argonautica de Valérius Flaccus (livres 1 et 2), Brüssel.

Stadler, H. 1993: Valerius Flaccus, Argonautica VII. Ein Kommentar, Hildesheim.

Strand, J. 1972: Notes on Valerius Flaccus' Argonautica, Göteborg.

Thilo, G. 1863: C. Valeri Flacci Argonauticon libri octo, Halle.

Venini, P. 1972: «Un propositi di un passo di Valerio Flacco (Arg. IV 148 ss.)», Athenaeum 50, pp. 404 - 406

Watt, W. S. 1984: «Notes on Latin Poetry», BICS 31, pp. 153-170.

Wijsman, H. J. W. 1996: Valerius Flaccus Argonautica Book V. A Commentary, Leiden.

- 2000: Valerius Flaccus Argonautica Book VI. A Commentary, Leiden.

Zissos, A. 2008: Valerius Flaccus' Argonautica Book I, Oxford.

Fecha de recepción de la primera versión del artículo: 02/10/2008

Fecha de recepción de la versión definitiva del artículo: 27/04/2010

Fecha de aceptación del artículo: 09/07/2009 